\title{
Deterioration without replenishment-the misery of oocyte cohesin
}

\author{
Rolf Jessberger ${ }^{1}$ \\ Institute of Physiological Chemistry, Faculty of Medicine Carl Gustav Carus, Dresden University of Technology, \\ D-01307 Dresden, Germany
}

\begin{abstract}
Humans suffer a steep increase in aneuploidies when oocytes age, and deterioration of cohesin was suggested recently as a prominent cause. In the November 15, 2010, issue of Genes \& Development, Tachibana-Konwalski and colleagues (pp. 2505-2516) answered a question central to this hypothesis: Can cohesin be reloaded onto mouse oocyte chromosomes long after birth? They found that it cannot, or at least not with an efficiency adequate to rescue cohesin deficiency. With no chance for sufficient replenishment, age-related loss of sister chromatid cohesion seems unavoidable.
\end{abstract}

In humans, many oocytes survive and wait for several decades to no avail: If called into action, a large fraction of oocytes fails to properly segregate their chromosomes and either dies or, rarely, survives with the potential to be fertilized and give rise to an aneuploid embryo. Several studies in recent years lend considerable support to the hypothesis of cohesin deterioration as a leading cause for chromosome missegregation in aging oocytes.

However, slow weakening or even disappearance of sister chromatid cohesion, which is facilitated by cohesin, would probably be prevented if cohesion could be reestablished by the reloading of cohesin onto chromosomes. In the November 15, 2010, issue of Genes \& Development, Kim Nasmyth and coworkers (Tachibana-Konwalski et al. 2010) addressed this key question of oocyte biology: Can cohesin be replenished and cohesion thereby be rescued? They showed that it cannot, or only insufficiently. Why is this lack of turnover of chromatid-associated cohesin so problematic and potentially very unfortunate for human beings?

The cohesin complex, which is essential for sister chromatid cohesion, consists of four subunits (for reviews, see Nasmyth 2005; Hirano 2006; Onn et al. 2008; Nasmyth and Haering 2009; Wood et al. 2010). Two of those proteins belong to the SMC (structural maintenance of chromosomes) family-SMC1 and SMC3 - and

[Keywords: Cohesin; Rec8; meiosis; oocytes]

${ }^{1}$ Correspondence.

E-MAIL rolf.jessberger@tu-dresden.de; FAX 49-0351-4586305.

Article is online at http://www.genesdev.org/cgi/doi/10.1101/gad.2000610. form a V-shaped heterodimer. Somatic cells and meiocytes (cells that proceed through meiosis) differ in the composition of their cohesin complexes. In somatic cells as well as in early stage meiocytes, the two SMC proteins SMC1 $\alpha$ and SMC3 associate with the kleisin RAD21/ SCC1/MDC1, such that the " $\mathrm{V}$ " is closed to form a ring. That ring-be it as a singular ring or as a more complex multiring module, such as a handcuff-like structureencircles the two sister chromatids and keeps them together until the metaphase-to-anaphase transition. To allow segregation of the sister chromatids, cleavage of the RAD21 subunit opens the ring, and the chromatids are released to move toward the opposite cell poles. The fourth cohesin protein is one of the SCC3-type proteins (either SA1 or SA2), which associate with RAD21 and may support sister chromatid cohesion, particularly at the telomeres and centromeres, respectively /Canudas and Smith 2009). SCC3 proteins may also be involved in the proposed association of two cohesin rings (Zhang et al. 2008). However, the functions of SCC3 proteins are not yet fully understood.

In vertebrate meiocytes, cohesin complexes are more varied than they are in somatic cells. Meiosis-specific cohesin proteins can replace the canonical ones: The meiotic kleisin REC8 can substitute for RAD21, STAG3 replaces $S A 1 / S A 2$, and $S M C 1 \beta$ replaces $S M C 1 \alpha$. It appears as if $\mathrm{SMC} 1 \alpha$ and $\mathrm{SMC} 1 \beta$ each form several different complexes with SMC3 and different non-SMC subunits. At the initial stages of meiosis, the SMCl $\alpha$-containing cohesin complex is still associated with the chromosomes and coimmunoprecipitates with either RAD21 or REC8. Similarly, SMC1 $\beta$ coimmunoprecipitates from testis nuclear extracts with REC8 or RAD21(Eijpe et al. 2000; Revenkova et al. 2004). The functional specialization of these distinct complexes in meiocytes are not known. Unlike the SMCl $\alpha$ based cohesin complex, SMC1 $\beta$-based cohesin is present in mouse spermatocytes and oocytes throughout meiosis up to the metaphase/anaphase II transition (Revenkova et al. 2001, 2004; Prieto et al. 2004). Like RAD21, the kleisin REC8 needs to be cleaved to allow meiotic chromosome segregation. Male mice expressing a noncleavable or cleavage-reduced variant of REC8, called REC8-N, are sterile. Their prophase I spermatocytes are normal, but instead of generating haploid spermatocytes II and spermatids, they were tetraploid. However, in oocytes, REC8-N 
is cleaved and the cells proceed through anaphase I, albeit with a delay (Kudo et al. 2009) .

Mouse strains deficient for REC8 and SMC1 $\beta$ were generated some years ago. Both deficiencies resulted in sterility of male and female mice, but caused quite distinct phenotypes. Besides displaying a deficiency in sister chromatid cohesion, $\operatorname{Rec} 8^{-7-}$ spermatocytes also showed abnormal synapsis, or pairing, between the sister chromatids, rather than between the pairs of sister chromatids, in the pachytene stage of prophase I. $\operatorname{Rec} 8^{-/-}$mice also suffered rapid loss of oocytes: They are almost completely absent by embryonic day 18.5 (i.e., approximately late pachytene) (Bannister et al. 2004; Xu et al. 2005), which precluded studies of oocyte aging in the REC8-deficient strain.

Such studies, however, were possible in $S m c 1 \beta^{-/-}$mice. While there is a progressive age-related loss of oocytes, cultured $S m c 1 \beta^{-/-}$oocytes can be matured in vitro until the metaphase II/anaphase II transition, when all cohesion is lost and the cells die (Revenkova et al. 2004; Hodges et al. 2005). Beyond $8 \mathrm{mo}$ of age, it is nearly impossible to detect oocytes in the $S m c 1 \beta^{-/-}$strain, but analysis of younger oocytes revealed important age-dependent phenotypes. Metaphase I chromosomes show a dramatic agedependent increase in the loss of bivalents; i.e., the two pairs of sister chromatids held together by chiasmata (Hodges et al. 2005). Loss of bivalents in Smc1 $\beta^{-/-}$oocytes strongly correlates with an increasingly distal localization and loss of chiasmata on metaphase I chromosomes. This suggested that, without SMC1 $\beta$-mediated sister chromatid cohesion, chiasmata cannot be held in place and cannot be prevented from terminalization-the falling off the edges of chromosomes and thus getting lost. Consistent with these observations, the shortest mouse chromosomes show the highest frequency of chiasmata loss. In metaphase II, SMC1 $\beta$ deficiency causes a complete loss of oocyte sister chromatid cohesion, illustrating that $\mathrm{SMC} 1 \beta$ is essential for continued meiotic sister chromatid cohesion. The endogenous SMCl $\alpha$ complex, which is initially present in meiocytes, appears to suffice only for initial prophase I cohesion, as it cannot rescue the later cohesion deficiency in $S m c 1 \beta^{-/-}$oocytes. The $S m c 1 \beta^{-/-}$ mouse was considered to reflect-albeit to a limited extent - the age-dependent increase in aneuploidies that is seen in humans (Bickel 2005; Gilliland and Hawley 2005; Hunt and Hassold 2010). This oocyte aging study was facilitated by the removal of one key cohesin in the presence of $\mathrm{SMCl} \alpha$, which provided for initial meiotic cohesion. But how do cohesins behave in mice that are not deficient in a cohesin?

In an aging-accelerated wild-type mouse strain, a lesser amount of REC8, STAG3, and SMC1 $\beta$ proteins was found on older oocyte chromosomes, which correlated with the premature separation of sister chromatids (Liu and Keefe 2008). As reported recently by the Lambson and Schultz groups (Chiang et al. 2010), the distance between sister chromatid centromeres increased up to $50 \%$ in aging wildtype mice. Metaphase I and metaphase II sister kinetochores in mouse oocytes of up to 19 mo of age were analyzed. The amount of chromosome-associated, but not of total, REC8 gradually decreased, while the incidence of aneu- ploidies increased in aging oocytes. Upon reduction of REC 8 to $<10 \%$ of the levels observed in 3 -mo-old mice, live imaging of oocytes progressing through anaphase I revealed a rapid increase of segregation errors. These data support the hypothetical link between weakening cohesion and increasing aneuploidies.

Similarly, the Herbert group (Lister et al. 2010) used a long-lived mouse strain to document an increase in interkinetochore distance in older oocytes. REC8 and chiasmata are increasingly lost from metaphase I chromosomes in these aging wild-type mice. Lister et al. (2010) also examined colocalization of REC8 and DNA in germinal vesicle stage oocytes, which is before nuclear envelope breakdown and progression through meiosis, and concluded that cohesion loss occurs while oocytes age when they are arrested in prophase I. Interestingly, an examination of the cohesin protector protein Sgo2 showed that it also progressively vanishes with increasing age.

Thus, evidence accumulates to support the cohesin deterioration hypothesis, as there indeed appears to be a loss of cohesin, and thus cohesion, with increasing age, and this strongly correlates with an increasing incidence of aneuploidies. However, can cohesin be reloaded onto chromosomes in aging oocytes? Would we, perhaps, suffer from much higher rates of aneuploidies if such reloading did not exist? Could one even wildly speculate about therapeutically reloading cohesin onto chromosomes in aging oocytes-a task quite likely to be highly dangerous and ethically questionable even if reloading is feasible? Or does this presumptive reloading process become inefficient in old oocytes, and thus its decline is responsible for the increased aneuploidies? Unless there exists a reserve of stable, unused, or recycled cohesin within the cell to be used years later, reloading would very likely require de novo cohesin synthesis during prophase arrest. Therefore, one question to ask is whether or not continuous cohesin expression is required to maintain cohesion in oocytes.

This question was answered by the removal of a critical cohesin gene right after birth: A conditional $S m c 1 \beta^{f l}$ allele (Revenkova et al. 2010) was excised within a few days after birth by a Gdf9 promoter-driven Cre recombinase. Although these Smc1 $\beta^{\mathrm{fl} / \mathrm{fl}}$ GDF9-Cre mice lack any source of newly synthesized SMC1 $\beta$ from dictyate arrest onward, they remain fully fertile well into old age, and their oocytes do not show any impairment of sister chromatid cohesion or chiasma maintenance and positioning up to 8 mo of age. In fact, cohesins were still present on metaphase I chromosomes many months after birth. All progeny of the Smc1 $\beta^{\mathrm{fl} / \mathrm{fl}}$ GDF9-Cre mice constitutively lacked $S m c 1 \beta$ expression, were sterile, and were phenotypically identical to the regular $\operatorname{Smc} 1 \beta^{-/-}$mice. While it became clear through this study that no newly synthesized cohesin is required in aging dictyate-arrested oocytes, the question for reloading of existing cohesin was not answered.

The cohesin decay hypothesis predicts that there would only be insufficient ways for the cell to re-establish cohesion with advancing age or, perhaps, no such mechanism at all. That prediction was tested in the recent study by Tachibana-Konwalski et al. (2010) in Genes \& Development. 
Nasmyth and colleagues (Tachibana-Konwalski et al. 2010) engineered mouse strains that express REC8 or SCC1 harboring target sites for the tobacco etch virus (TEV) protease. Microinjection of $\operatorname{Rec} 8^{\text {TEV/TEV }}$ or $S c c 1^{\text {TEV/TEV }}$ oocytes with TEV mRNA induces cleavage and the efficient elimination of REC8- or SCC1-type cohesins. TEV-mediated cleavage of REC8 in $\operatorname{Rec} 8^{\text {TEV/TEV }}$ oocytes was sufficient for chiasma resolution even when separase was inhibited through the overexpression of MAD2 and subsequent suppression of securin degradation. Microinjection of TEV mRNA into Rec $8^{T E V / T E V}$ germinal vesicle oocytes caused the complete separation of sister chromatids (i.e., loss of cohesion and bivalents), demonstrating both the efficiency and functional consequences of TEV cleavage. The bivalent structure-that is, the synapsis of the two pairs of sister chromatids-was not affected by TEV expression in Scc1 $1^{\text {TEVMYC/TEVMyc }}$ oocytes. TEV expression in metaphase II-arrested $\operatorname{Rec} 8^{\text {TEV/TEV }}$ oocytes caused disjunction of sister centromeres. There was no effect on centromeric sister chromatid cohesion if $S c c 1^{\text {TEVMyc/TEVMyc }}$ oocytes were treated, demonstrating the importance and specificity of REC8 in oocytes. Why, however, is SCC1 abundantly expressed in oocytes? If $\mathrm{SCC} 1^{\mathrm{TEVMMc} / \mathrm{TEVMMc}}$ is cleaved by TEV in zygotes, the first embryonic cell division is severely affected: Loss of sister chromatid cohesion is seen in metaphase. However, cleavage of REC $8^{\mathrm{TEV} / \mathrm{TEV}}$ had no effect on embryonic cell division. This suggests that a rapid switch occurs: When embryonic life commences, REC8-dependent and SCC1independent cohesion switches to REC8-independent, SCC1-dependent cohesion. The requirement for the canonical, somatic cohesin to be ready for use in the emerging embryo may also explain the presence of $S m c 1 \alpha$ transcripts in growing oocytes (R Jessberger, unpubl.). Tachibana-Konwalski et al. (2010) thus showed that REC8-type cohesin is necessary and sufficient for both arm and centromere cohesion in oocytes. But what about the putative reloading of cohesin?
The Nasmyth group (Tachibana-Konwalski et al. 2010) further developed their experimental system by adding a stage-specific REC8 expression cassette in which a stop codon can be removed through the Cre-mediated cleavage of loxP sites. Either the Sox2 promoter or the $\mathrm{Zp} 3$ promoter drives the expression of Cre, which induces expression of a myc-tagged REC8 either in premeiotic germ cells or 2-3 wk after birth (in the growing oocyte), respectively. This allowed Tachibana-Konwalski et al. (2010) to test whether expression of REC8-myc complements the TEV-induced REC8 deficiency, which would require loading onto chromosomes. However, expression of REC8-myc prevented the loss of cohesion only if it was expressed before entry into meiosis; i.e., if REC8-myc was present when cohesion was established at the entry into meiosis. This also proved functionality of the REC8-myc protein. Expression of REC8-myc in growing oocytes a few weeks after the birth of the female mice did not compensate for the loss of REC $8^{\mathrm{TEV} / \mathrm{TEV}}$. Thus, there is either none or an insufficient reloading of functional REC8-cohesin in this system. These important results strongly support the hypothesis of cohesin deterioration, which calls for no or too little replenishment of cohesin on oocyte chromosomes.

Although it has thus been shown that cohesin produced before birth is sufficient for cohesion even in aging oocytes (Revenkova et al. 2010), and while there is little or no cohesin reloading (Tachibana-Konwalski et al. 2010), one cannot yet exclude the possibility of a low rate of reloading from a pool of mRNA or protein that exists since before birth. However, whether such a pooled reserve of cohesin can survive for more than a year in mice is questionable. Since a low level of Smc1 $\beta$ mRNA has been observed in postnatal mouse oocytes (Hodges et al. 2005) and other cohesin genes may also be lowly transcribed, it is possible that limited cohesin protein synthesis may take place. However, the new data suggest that whatever synthesis occurs is likely insufficient and may never be loaded onto

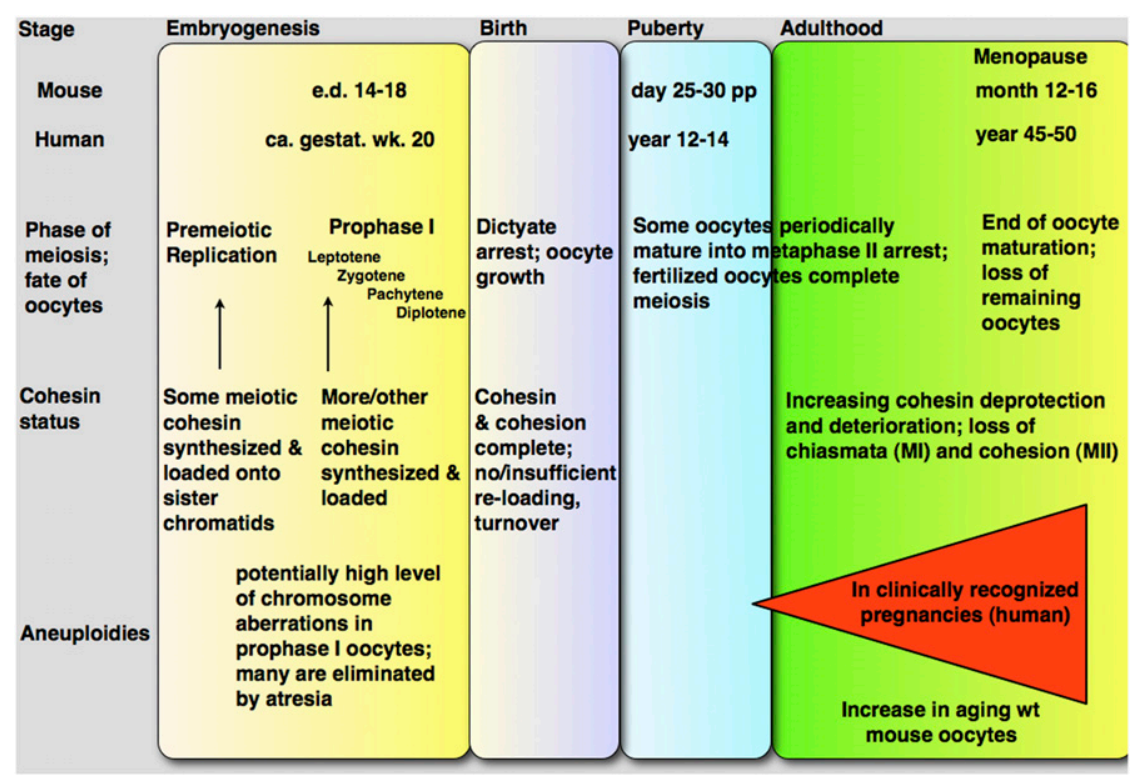

Figure 1. Summary of oocyte development, cohesin status, and aneuploidies. (Top) Stages of human and mouse life are correlated with phases of meiosis, the status of cohesin, and the level of aneuploidies. A red triangle illustrates the rapid increase in aneuploidies during human female aging, particularly beyond $35 \mathrm{yr}$ of age. Timing of embryonic prophase I, puberty, and menopause is approximate, and, in mice, depends on the particular mouse strain. The exact timing of onset of expression of each individual cohesin protein has not yet been determined, but indications exist for post-replicative loading of some cohesin in early prophase I. It is also unknown when after or even before birth cohesin deterioration starts. (Gestat. wk.) Gestational week; (e.d.) embryonic day. 
chromosomes. That insufficiency also became apparent in the above-described studies on aging wild-type mice (Chiang et al. 2010; Lister et al. 2010). Complete absence of, or too little, cohesin loading after birth prevents the cell from properly counteracting the slow degradation of cohesin, and thus from counteracting the rise in aneuploidies.

The jury is still out as to the physiological relevance of this mouse data to humans. How do cohesins behave in human oocytes? The scarcity of differently aged material and the inability to manipulate these oocytes have hampered such studies. Recently, the abundance and localization of some cohesin proteins-including REC8, STAG3, and SMC1 $\beta$, as well as SMC3 - together with components of the synaptonemal complex, were investigated in human oocytes representing several stages of meiosis (Garcia-Cruz et al. 2010). In agreement with data from mouse studies, these cohesins localize to centromeres, along chromosome arms, and at each side of chiasmata of metaphase I chromosomes. As expected from mouse data, the cohesins only localized to the centromeres from anaphase I to metaphase II, and disappeared thereafter. Garcia-Cruz et al. (2010) quantified $S m c 1 \beta$ transcripts from germinal vesicle-stage oocytes from women 19-43 yr of age, but high variability between samples precluded a clear conclusion with respect to a correlation with age. Realtime PCR measurements of $S m c 1 \beta$ mRNA revealed that the transcripts remain in mouse oocytes at least up to 6 mo of age, but at levels that are $<10 \%$ of those found in embryonic day 18 oocytes (Hodges et al. 2005). Ultimately, whether in humans or in mice, if there is no cohesin reloading, transcription would not help. "Why, then, transcribe at all?" is an open question.

While the evidence in support of cohesin deterioration as a leading cause of age-related aneuploidies has became considerably stronger through this year's studies, many questions remain to be addressed (Fig. 1). Would massive, simultaneous expression of all cohesin subunits force loading of new cohesin onto chromosomes, and would this be sufficient to complement cohesin deterioration? How many cohesin rings are required to keep sister chromatids together, at least at their centromeres, or to keep chiasma in place to maintain bivalents? Is there indeed a critical threshold level of cohesin per chromosome that is required for proper cohesion, as some of the very recent studies suggest? The exponential increase of aneuploidies seen in mouse oocytes beyond a certain age, and in human embryos with advancing age of the mother, seems to suggest a rapid deterioration of cohesion, once cohesin numbers have fallen below some minimum. Perhaps most importantly, however, is the question as to why cohesin deteriorates. The disappearance of Sgo2, as important as this observation is, is not a satisfying answer, as it triggers the question of why that happens to Sgo2. In fact, it is unknown whether the decrease in Sgo2 is a cause or consequence of losing cohesin at centromeres, since degradation may happen anywhere in the ring. Furthermore, how stable can a chromosome-associated, multisubunit protein complex be? How stable must it be to survive up to five decades unharmed? If one single break in the ring is sufficient to destroy sister chromatid cohesion, then it is not unlikely that even spontaneous hydrolysis of one of the cohesin proteins may be at the root of the problem.

\section{Acknowledgments}

Work in our laboratory is supported by a grant from the DFG (SPP1384; JE 150/10-1).

\section{References}

Bannister LA, Reinholdt LG, Munroe RJ, Schimenti JC. 2004. Positional cloning and characterization of mouse mei8, a disrupted allelle of the meiotic cohesin Rec8. Genesis 40: 184-194.

Bickel SE. 2005. Aging (not so) gracefully. Nat Genet 37: 13031304.

Canudas S, Smith S. 2009. Differential regulation of telomere and centromere cohesion by the Scc3 homologues SA1 and SA2, respectively, in human cells. J Cell Biol 187: 165-173.

Chiang T, Duncan FE, Schindler K, Schultz RM, Lampson MA. 2010. Evidence that weakened centromere cohesion is a leading cause of age-related aneuploidy in oocytes. Curr Biol 20: $1522-1528$.

Eijpe M, Heyting C, Gross B, Jessberger R. 2000. Association of mammalian SMC1 and SMC3 proteins with meiotic chromosomes and synaptonemal complexes. J Cell Sci 113: 673682.

Garcia-Cruz R, Brieno MA, Roig I, Grossmann M, Velilla E, Pujol A, Cabero L, Pessarrodona A, Barbero JL, Garcia Caldes M. 2010. Dynamics of cohesin proteins REC8, STAG3, SMC1 $\beta$ and SMC3 are consistent with a role in sister chromatid cohesion during meiosis in human oocytes. Hum Reprod 25: 2316-2327.

Gilliland WD, Hawley RS. 2005. Cohesin and the maternal age effect. Cell 123: 371-373.

Hirano T. 2006. At the heart of the chromosome: SMC proteins in action. Nat Rev Mol Cell Biol 7: 311-322.

Hodges CA, Revenkova E, Jessberger R, Hassold TJ, Hunt PA. 2005. SMC1 $\beta$-deficient female mice provide evidence that cohesins are a missing link in age-related nondisjunction. Nat Genet 37: 1351-1355.

Hunt P, Hassold T. 2010. Female meiosis: Coming unglued with age. Curr Biol 20: R699-R702. doi: 10.1016/j.cub.2010.08.011.

Kudo NR, Anger M, Peters AH, Stemmann O, Theussl HC, Helmhart W, Kudo H, Heyting C, Nasmyth K. 2009. Role of cleavage by separase of the Rec8 kleisin subunit of cohesin during mammalian meiosis I. J Cell Sci 122: 2686-2698.

Lister LM, Kouznetsova A, Hyslop LA, Kalleas D, Pace SL, Barel JC, Nathan A, Floros V, Adelfalk C, Watanabe Y, et al. 2010. Age-related meiotic segregation errors in Mammalian oocytes are preceded by depletion of cohesin and sgo2. Curr Biol 20: 1511-1521.

Liu L, Keefe DL. 2008. Defective cohesin is associated with agedependent misaligned chromosomes in oocytes. Reprod Biomed Online 16: 103-112.

Nasmyth K. 2005. How might cohesin hold sister chromatids together? Philos Trans R Soc Lond B Biol Sci 360: 483-496.

Nasmyth K, Haering CH. 2009. Cohesin: Its roles and mechanisms. Annu Rev Genet 43: 525-558.

Onn I, Heidinger-Pauli JM, Guacci V, Unal E, Koshland DE. 2008. Sister chromatid cohesion: A simple concept with a complex reality. Annu Rev Cell Dev Biol 24: 105-129.

Prieto I, Tease C, Pezzi N, Buesa JM, Ortega S, Kremer L, Martinez A, Martinez AC, Hulten MA, Barbero JL. 2004. 
Cohesin component dynamics during meiotic prophase I in mammalian oocytes. Chromosome Res 12: 197-213.

Revenkova E, Eijpe M, Heyting C, Gross B, Jessberger R. 2001. Novel meiosis-specific isoform of mammalian SMC1. Mol Cell Biol 21: 6984-6998.

Revenkova E, Eijpe M, Heyting C, Hodges CA, Hunt PA, Liebe B, Scherthan H, Jessberger R. 2004. Cohesin SMC1 $\beta$ is required for meiotic chromosome dynamics, sister chromatid cohesion and DNA recombination. Nat Cell Biol 6: 555-562.

Revenkova E, Herrmann K, Adelfalk C, Jessberger R. 2010. Oocyte cohesin expression restricted to predictyate stages provides full fertility and prevents aneuploidy. Curr Biol 20: 1529-1533.

Tachibana-Konwalski K, Godwin J, van der Weyden L, Champion L, Kudo NR, Adams DJ, Nasmyth K. 2010. Rec8-containing cohesin maintains bivalents without turnover during the growing phase of mouse oocytes. Genes Dev 24: 2505-2516.

Wood AJ, Severson AF, Meyer BJ. 2010. Condensin and cohesin complexity: The expanding repertoire of functions. Nat ReV Genet 11: 391-404.

Xu H, Beasley MD, Warren WD, van der Horst GT, McKay MJ. 2005. Absence of mouse REC8 cohesin promotes synapsis of sister chromatids in meiosis. Dev Cell 8: 949-961.

Zhang N, Kuznetsov SG, Sharan SK, Li K, Rao PH, Pati D. 2008. A handcuff model for the cohesin complex. J Cell Biol 183: 1019-1031. 


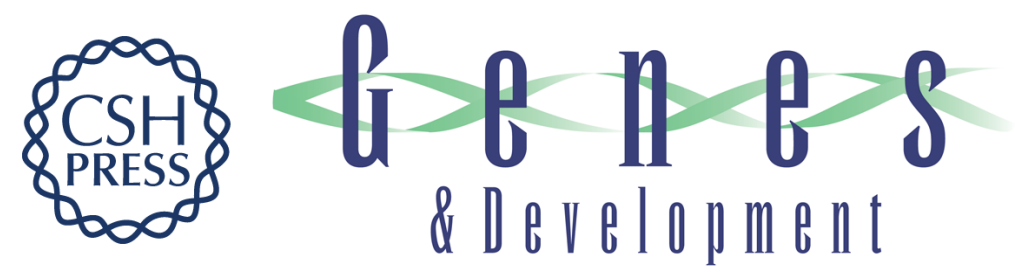

\section{Deterioration without replenishment--the misery of oocyte cohesin}

Rolf Jessberger

Genes Dev. 2010, 24:

Access the most recent version at doi:10.1101/gad.2000610

Related Content Rec8-containing cohesin maintains bivalents without turnover during the growing phase of mouse oocytes

Kikuë Tachibana-Konwalski, Jonathan Godwin, Louise van der Weyden, et al.

Genes Dev. November , 2010 24: 2505-2516

References This article cites 24 articles, 6 of which can be accessed free at:

http://genesdev.cshlp.org/content/24/23/2587.full.html\#ref-list-1

Articles cited in:

http://genesdev.cshlp.org/content/24/23/2587.full.html\#related-urls

\section{License}

Email Alerting Receive free email alerts when new articles cite this article - sign up in the box at the top Service right corner of the article or click here.

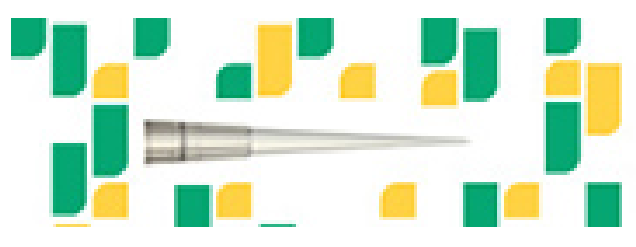

Focused on your science. 